\title{
Cryptococcal meningitis mimicking cerebral infarction: a case report
}

This article was published in the following Dove Press journal:

Clinical Interventions in Aging

\author{
Weihua Zhou ${ }^{1-3, *}$ \\ Jianbo Lail-3,* \\ Tingting Huang ${ }^{\prime}$ \\ Yi Xu ${ }^{1-3}$ \\ Shaohua $\mathrm{Hu}^{1-3}$ \\ 'Department of Psychiatry, The First \\ Affiliated Hospital, Zhejiang University \\ School of Medicine, Hangzhou 310003, \\ China; ${ }^{2}$ The Key Laboratory of Mental \\ Disorder's Management in Zhejiang \\ Province, Hangzhou 310003, China; \\ ${ }^{3}$ Brain Research Institute of Zhejiang \\ University, Hangzhou 3I0003, China \\ *These authors contributed equally \\ to this work
}

\begin{abstract}
Cryptococcal meningitis (CM) is the most common type of fungal meningitis. The clinical symptoms of $\mathrm{CM}$ are nonspecific, and neuroimaging characteristics are variable. Herein, we present a case of a senile female with CM that was once misdiagnosed as cerebral infarction. Her condition worsened and she developed hydrocephalus. No apparent predisposing factors of $\mathrm{CM}$ were reported in this patient. The diagnosis of $\mathrm{CM}$ was definitely made after India ink staining of cerebrospinal fluid was positive. This case indicates that clinicians should bear cryptococcal infection in mind when the symptoms are nonspecific and neuroimaging findings are atypical.
\end{abstract}

Keywords: cryptococcal meningitis, cerebral infarction, neuroimaging

\section{Introduction}

Cryptococcal meningitis $(\mathrm{CM})$, predominantly caused by intracranial infection by Cryptococcus neoformans or Cryptococcus gattii, is the most common type of fungal meningitis in humans. ${ }^{1}$ The incidence of $\mathrm{CM}$ has increased in recent decades, especially in immunocompromised patients. ${ }^{2}$ Predisposing factors for cryptococcal infection include diabetes, cancer, HIV infection, chemotherapeutic agents, corticosteroid therapy, renal failure/dialysis, lung diseases, and hematological malignancies. ${ }^{2} \mathrm{CM}$ is usually developed in a chronic or subacute course and manifests as headache, fever, vomiting, altered consciousness, seizure, visual loss, behavior changes, and cognitive impairment. ${ }^{3}$ The identification of Cryptococcus in the cerebrospinal fluid (CSF) or cryptococcal antigen is sufficient for diagnosis. Neuroimaging examinations are necessary in patients suspected of CM before carrying out lumbar puncture. ${ }^{3}$ Cranial magnetic resonance imaging (MRI) or computed tomography (CT) scanning helps to differentiate $\mathrm{CM}$ from other diseases such as tuberculous meningitis, viral meningitis, encephalopyosis, and glioma. The typical characteristics of neuroimaging alterations included dilated Virchow-Robin (V-R) spaces, pseudocyst, cryptococcomas, and leptomeningeal enhancement., ${ }^{4,5}$

However, the clinical pictures of $\mathrm{CM}$ are usually nonspecific. Moreover, neuroimaging reports of $\mathrm{CM}$ in immunocompetent individuals are limited. ${ }^{4,5}$ These factors contribute to the false and/or delayed diagnosis of this disease in a substantial proportion of patients. Herein, we present a case of an old woman without any known predisposing factor, who encountered $\mathrm{CM}$ and was once misdiagnosed as cerebral infarction.

\section{Case presentation}

Mrs A, an 81-year-old woman, was admitted to the Department of Infectious Diseases of The First Affiliated Hospital, Zhejiang University School of Medicine because of 
disturbance in her level of consciousness. Before this admission, the patient was diagnosed with $\mathrm{CM}$ in a neighboring hospital, where the diagnosis of CM was made after finding Cryptococcus in the CSF, and she underwent ventriculopuncture and implantation of Ommaya reservoir because of severe hydrocephalus. However, the patient had been previously diagnosed with cerebral infarction before diagnosing CM.

Nearly half a year prior to this admission, the patient ventured out and cut firewood alone after quarreling with her family members. Three days later, she began to feel headache, muscle soreness, and fatigue. Her condition worsened in the following days and she felt extremely weak and even could not walk or eat by herself. The patient was then sent to a local hospital. Cranial MRI revealed multiple ischemic foci located in the junction of cortex and medulla of bilateral frontoparietal region, the periventricular area, and the centrum semiovale. Cranial magnetic resonance angiography (MRA) revealed regional stenosis in the A1 segment of the left anterior cerebral artery and the P2 segment of the right posterior cerebral artery. Carotid artery ultrasound reported multiple plaques in the right common carotid artery with appropriately $79 \%$ stenosis. Systemic and supportive treatment did not help to improve her condition. The patient still complained of extreme weakness and poor appetite. Four weeks later, another cranial MRI indicated new-onset lacunar infarction in the bilateral frontal lobe. Cranial CT indicated high-density image near the anterior horn of the right lateral ventricle. Four months before this admission, the patient developed disturbance in her level of consciousness and psychotic symptoms, including persecutory delusion and visual hallucination. Her weight had decreased by $3.5 \mathrm{~kg}$ since she fell ill. She was then admitted to the Department of Psychiatry of our hospital. At that time, the patient failed to cooperate with physical examination and psychiatric interview. No pathological reflection of Babinski sign was induced. Occasionally, she would have incontinence of feces and urine. She reported congenital absence of left kidney and uterus duplex. No history of hypertension, diabetes mellitus, heart disease, or tuberculosis was reported. She did not drink or smoke or have long-term medications. Laboratory examinations, including arterial blood gas analysis; routine tests of blood, stool, and urine; liver and kidney functions; thyroid hormones; tumor biomarkers; myocardial enzymes; infectious profiles of HIV; syphilis; and hepatitis B and C, were all within normal limits. The serum D-dimer level was extremely high, and the potassium level was a bit low. The third cranial MRI indicated acute cerebral infarction in the bilateral frontoparietal region and ischemic changes in the periventricular area and the centrum semiovale (Figures 1 and 2). Thoracic CT revealed scattered infectious loci in the bilateral lungs. Neurological consultation was obtained, and the diagnosis was considered to be cerebral infarction. However, the patient was physically weak and uncooperative, and lumbar puncture was not performed due to the potential risk. Nutritional support and symptomatic treatment were prescribed, but the outcome was unsatisfactory. Her psychotic symptoms were well controlled by low-dose of quetiapine (50 mg per night). The patient was then transferred to a local hospital for further rehabilitation.

However, her condition did not improve. She raved occasionally and complained of headache and weakness. Cranial CT reported patch-shaped low-density areas around the bilateral periventricular region, which indicated hydrocephalus. Her illness further worsened in the following days and she developed lethargy, conscious disturbance, and retching. Consequently, she was transferred to a neighboring hospital to ours. An enhanced cranial MRI scanning indicated supratentorial obstructive hydrocephalus and interstitial encephaledema in bilateral periventricular region. Lumbar puncture was performed, and examination of CSF indicated cryptococcal antigen (+), India ink stain $(+)$, chlorine $109.1 \mathrm{mmol} / \mathrm{L}(\downarrow)$, glucose $0.1 \mathrm{mmol} / \mathrm{L}(\downarrow)$, protein $336.5 \mathrm{mg} / \mathrm{dL}(\uparrow)$, karyocyte count $24 \times 10^{6} / \mathrm{L}$, and lymphocyte (98\%). She was diagnosed with CM, received ventriculopuncture and implantation of Ommaya reservoir, and initiated antifungal interventions. Five days later, she was sent to the Department of Infectious Diseases of our hospital for further treatment. Given the potential renal toxicity of amphotericin B and absence of left kidney in our patient, $800 \mathrm{mg}$ fluconazole per day was used for induction therapy against $C$. neoformans. Mannitol was used to reduce intracranial pressure. The patient began to show clinical improvement under intensive nursing and management. Three weeks after admission, lumbar puncture was performed again, and no Cryptococcus was found in the CSF. The patient was then discharged from our hospital, and her condition further improved in the follow-ups.

This case study was approved by the institute ethical committee of the First Affiliated Hospital, Zhejiang University School of Medicine. Written informed consent was obtained from the guardians of this patient to publish the case details.

\section{Discussion}

In this case study, we report a case of chronic CM misdiagnosed as cerebral infarction in an immune-competent female patient. No evident predisposing factors for CM have been 

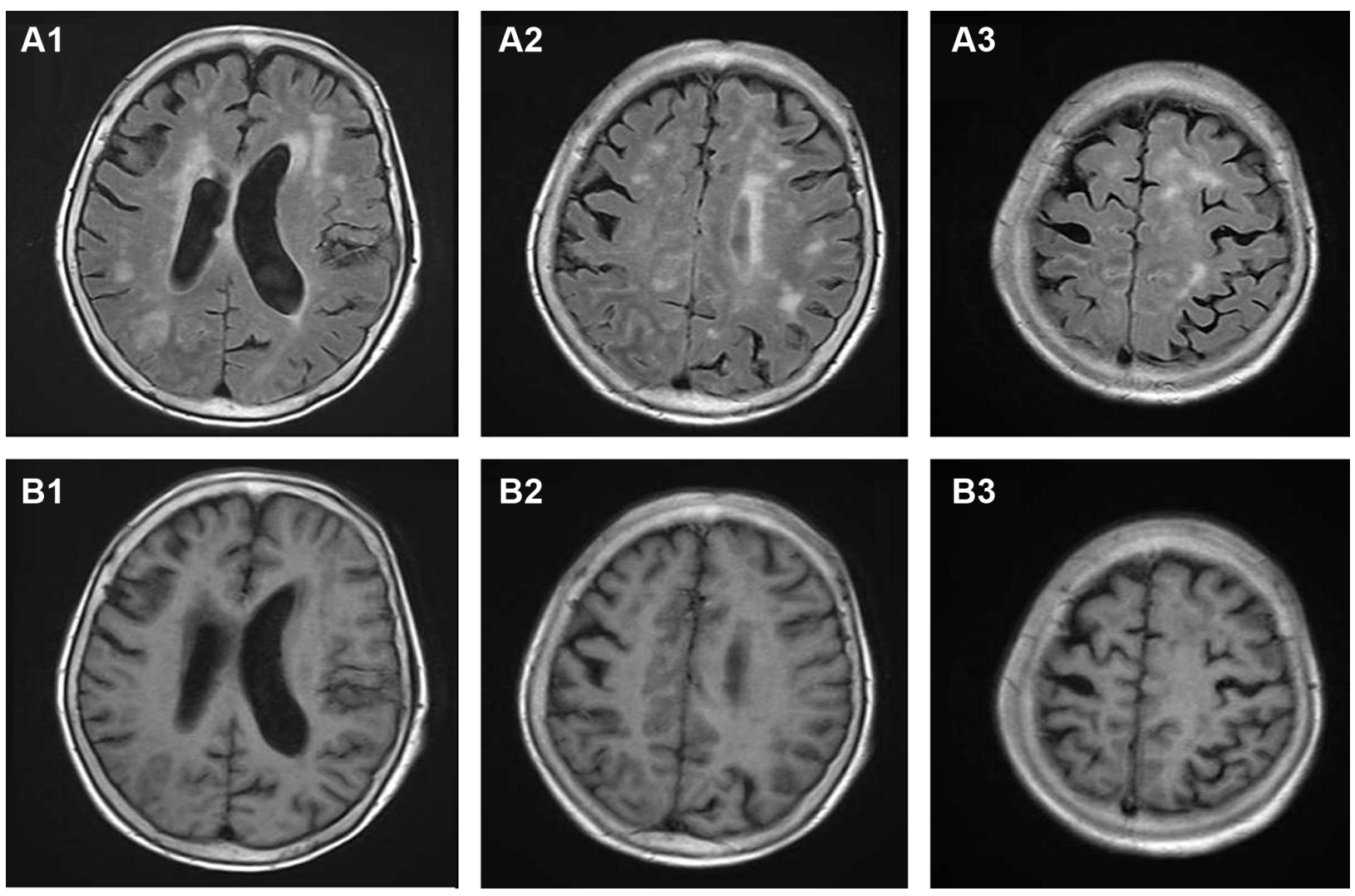

Figure I Cranial MRI scanning of the patient.

Notes: (AI-3) High T2-FLAIR signal on different sections by transection plane scan. (B I-3) Low TI-FLAIR signal on different sections by transection plane scan. Abbreviations: MRI, magnetic resonance imaging; FLAIR, fluid-attenuated inversion-recovery sequence.

discovered in this old patient. However, her experience of chopping firewood before getting sick could possibly weaken her resistance to cryptococcal infection. The false diagnosis of cerebral infarction was likely related to following causes.

The clinical symptoms of CM are nonspecific. Our patient had no fever during the illness course. Her predominant manifestation was headache. As previously reported, headache is the most common clinical manifestation of $\mathrm{CM}$,
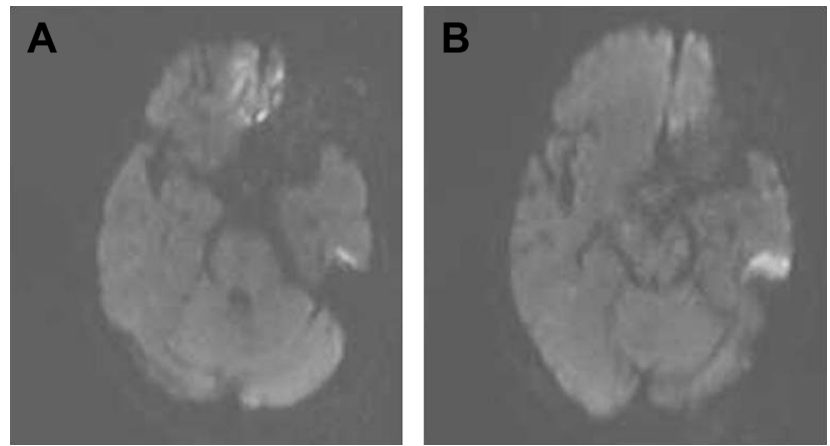

Figure 2 Cranial MRI scanning indicating high DWI signal.

Note: (A) Bilateral frontal lobe; (B) left temporal lobe.

Abbreviations: MRI, magnetic resonance imaging; DWI, diffusion-weighted imaging. which may result from increased intracranial pressure and can present for weeks or even months. However, fever only exists in nearly half of all patients. ${ }^{3}$ The absence of fever would possibly dismiss the diagnosis of infectious diseases. Other symptoms in our patient, such as weakness, inability to walk, psychotic symptoms, lethargy, and conscious disturbance, could also be encountered by patients with cerebral infarction. Moreover, common peripheral blood tests, such as routine examinations of blood, urine and stool, biochemical profiles, and C-reactive protein, cannot indicate the infection of fungal infection. In recent years, an immune assay testing the cryptococcal antigen in serum and CSF has been approved by the US Food and Drug Administration (FDA). Unfortunately, although lung infection had been demonstrated by CT scanning in our patient, the causative pathogen was not further determined. Lumbar puncture was not performed during the first admission in our hospital, which resulted in the missed diagnosis of CM.

Knowledge on the neuroimaging alterations of $\mathrm{CM}$ is insufficient for many clinicians. Studies on neuroimaging findings in immunocompetent patients are also limited. ${ }^{4-6}$ Multiple dilated V-R spaces, gelatinous pseudocysts, 
hydrocephalus, and leptomeningeal enhancement are the common neuroimaging characteristics of CM..${ }^{4-6}$ Dilated V-R spaces are generated from the accumulation of Cryptococcus and voluminous inflammatory exudates in the perivascular spaces. However, MRI features of dilated V-R spaces are similar to lacunar infarction and located mainly in the basal ganglia, centrum semiovale, corpus callosum, cerebellum, and brainstem. ${ }^{5}$ Fusion of dilated V-R spaces forms gelatinous pseudocysts. In our patient, cranial MRI reported multiple ischemic loci in the bilateral frontoparietal region, the periventricular area, and centrum semiovale. Carotid artery ultrasound and cranial MRA revealed severe arterial stenosis, which was considered as the cause of cerebral ischemia. Neurological consulting also suggested the diagnosis of cerebral infarction. Although this patient had no hypertension, diabetes, and heart diseases, her risk of stroke (score 3) evaluated by Essen Stroke Risk Scale was moderate. ${ }^{7}$ However, the condition of our patient deteriorated when she was treated for ischemic cerebrovascular disease.

Notably, it was also possible that our patient had both $\mathrm{CM}$ and cerebral infarction. It has been reported that acute/ subacute cerebral infarction could occur in patients with $\mathrm{CM}^{8,9}$ and predominantly located in the basal ganglia, thalamus, frontal, temporal, and parieto-occipital regions. ${ }^{10}$ Cerebrovascular injury, such as vasculitis, vasospasm, and thrombosis, could contribute to the occurrence of cerebral infarction in CM patients. ${ }^{5,10} \mathrm{~A}$ recent case report indicated that subarachnoid small vein occlusion caused by inflammatory fibrosis was also a possible mechanism for cerebral infarction in CM. ${ }^{11}$ In our patient, acute cerebral infarction in the bilateral frontoparietal region had been revealed by cranial MRI scanning. However, no apparent lesions of cerebral infarction were observed in the next enhanced MRI scanning. This improvement in neuroimaging might indicate that the cerebral infarction was caused by Cryptococcus.

\section{Conclusion}

We herein report a case of CM mimicking cerebral infarction. The missed diagnosis of $\mathrm{CM}$ and delayed treatment led to the deterioration of symptoms and the formation of hydrocephalus. Clinicians should consider the possible infection of Cryptococcus when the symptoms are nonspecific and patterns of cerebral infarction are atypical on cranial imaging data.

\section{Acknowledgments}

This study was supported by grants from the National Key Research and Development Program (2016YFC1307100 and 2016YFC1307102), the National Science and Technology Program (2015BAI13B02), and the Key Research Project of Zhejiang Province (2015C03040). We thanked the patient and her family for their support and approval for publishing the case.

\section{Disclosure}

The authors report no conflicts of interest in this work.

\section{References}

1. Makadzange AT, Mchugh G. New approaches to the diagnosis and treatment of cryptococcal meningitis. Semin Neurol. 2014;34(1):47-60.

2. Góralska K, Blaszkowska J, Dzikowiec M. Neuroinfections caused by fungi. Infection. 2018;46(4):443-459.

3. Satishchandra P, Mathew T, Gadre G, et al. Cryptococcal meningitis: clinical, diagnostic and therapeutic overviews. Neurol India. 2007; 55(3):226-232.

4. Tan ZR, Long XY, Li GL, Zhou JX, Long L. Spectrum of neuroimaging findings in cryptococcal meningitis in immunocompetent patients in China - A series of 18 cases. J Neurol Sci. 2016;368:132-137.

5. Chen S, Chen X, Zhang Z, Quan L, Kuang S, Luo X. MRI findings of cerebral cryptococcosis in immunocompetent patients. J Med Imaging Radiat Oncol. 2011;55(1):52-57.

6. Sarkis RA, Mays M, Isada C, Ahmed M. MRI findings in cryptococcal meningitis of the non-HIV population. Neurologist. 2015;19(2): $40-45$.

7. Diener HC, Ringleb PA, Savi P. Clopidogrel for the secondary prevention of stroke. Expert Opin Pharmacother. 2005;6(5):755-764.

8. Chen YF, Wang DN, Chen ZT, et al. Risk factors associated with acute/ subacute cerebral infarction in HIV-negative patients with cryptococcal meningitis. J Neurol Sci. 2016;364:19-23.

9. Lan SH, Chang WN, Lu CH, Lui CC, Chang HW. Cerebral infarction in chronic meningitis: a comparison of tuberculous meningitis and cryptococcal meningitis. QJM. 2001;94(5):247-253.

10. Mishra AK, Arvind VH, Muliyil D, et al. Cerebrovascular injury in cryptococcal meningitis. Int J Stroke. 2018;13(1):57-65.

11. Shimoda Y, Ohtomo S, Arai H, Ohtoh T, Tominaga T. Subarachnoid small vein occlusion due to inflammatory fibrosis-a possible mechanism for cerebellar infarction in cryptococcal meningoencephalitis: a case report. BMC Neurol. 2017;17(1):157.
Clinical Interventions in Aging

\section{Publish your work in this journal}

Clinical Interventions in Aging is an international, peer-reviewed journal focusing on evidence-based reports on the value or lack thereof of treatments intended to prevent or delay the onset of maladaptive correlates of aging in human beings. This journal is indexed on PubMed Central, MedLine,

\section{Dovepress}

CAS, Scopus and the Elsevier Bibliographic databases. The manuscript management system is completely online and includes a very quick and fair peer-review system, which is all easy to use. Visit http://www.dovepress. com/testimonials.php to read real quotes from published authors. 